\title{
A GuIde To USING MATERIAL MODEL \#11 IN NIKE2D: AN INTERNAL VARIABLE, VISCOPLASTICITY MODEL*
}

\author{
Elane C. Flower and Daniel J. Nikkel, Jr. \\ Mechanics of Materials Group \\ Engineering Science Division \\ Lawrence Livermore National Laboratory
}

\begin{abstract}
The need to accurately model the superplastic forming process which is highly rate and temperature dependent motivated the evaluation of Bammann's internal variable, viscoplasticity material model. The model is based upon the concepts of unified creep plasticity, but employs a yield surface for efficient implementation into large-scale numerical computer codes. It has proven elsewhere to be quite successful in describing large strain, thermal-mechanical behavior of crystalline materials. Features of the model enable it to simulate the apparent strain-rate behavior exhibited by many metals above one half the melt temperature. It is the efficient incorporation of these features that make the model attractive for use in finite element modeling of metal deformation processes. Although this model was implemented into the Lawrence Livermore National Laboratory's NIKE2D finite element program in 1986, there have been no known reports of successful use by NIKE2D users. The purpose of this report is to provide the user the proper format to input model parameters, a procedure for determining appropriate values for material constants from experimental data, and supplemental information on the model relevant to the implementation in the NIKE2D finite element program. Detailed accounts of the theoretical aspects of the model can be found in the cited references.
\end{abstract}




\section{CONTENTS}

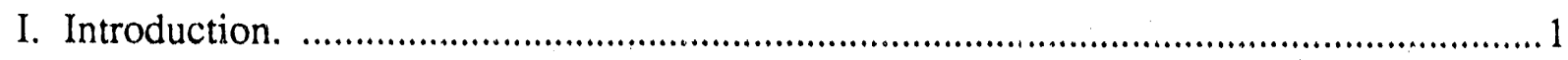

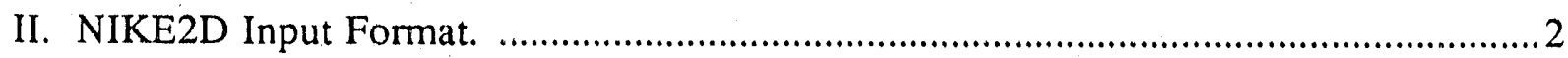

III. Discussion of Material Constants in Bammann's Model. .......................................... 3

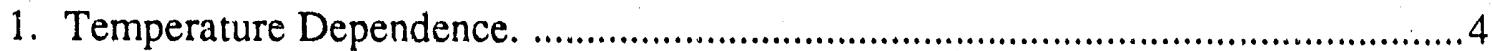

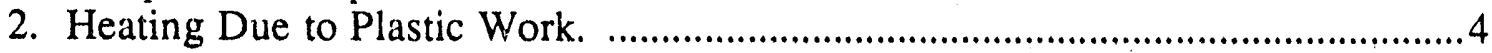

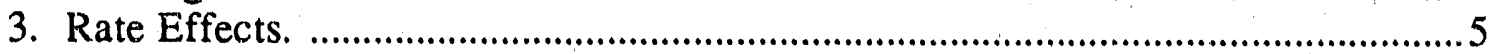

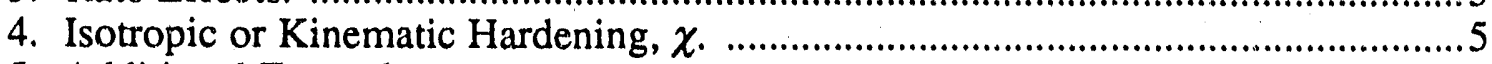

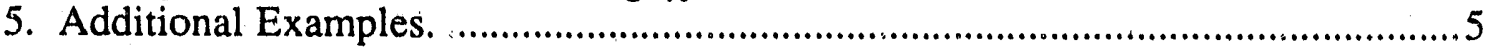

IV. Determining Values for the Material Constants. ...................................................6

1. Determination of Values of the Parameters from Experimental Data. .................6

2. Determination of Constants from Known Values of the Parameters. ……...........9

V. An Example Set of Material Constants for Model \#11. ..............................................10

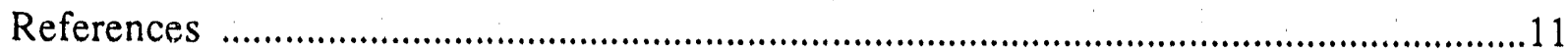

Appendix A. Basic Equations of Bammann's Model. ...................................................12

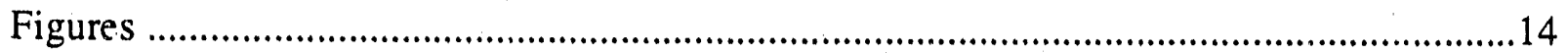

\section{Introduction.}

A rate and temperature dependent plasticity model developed by Bammann (1) was implemented in 1986 into the NIKE2D (2) finite element program as model \#11, "Unified Creep Plasticity", while a more accurate name for the model would be "Rate and Temperature Dependent Plasticity". Although it has been available for several years in NIKE2D, there has been no reported use or evaluation of this material model. Bammann's model is similar in many ways to the class of unified creep plasticity models that are formulated without the introduction of a yield surface. In contrast, Bammann's model incorporates a viscoplastic yield surface whose size and evolution depend upon a set of appropriate internal variables. The basic assumption in the model is that inelastic flow depends upon the value of stress and current value of two internal variables, a scalar and a tensor. The evolution of the internal variables is formulated in the "hardening minus recovery format". The model also differentiates between rate sensitivity of the yield stress and the rate sensitivity of a particular recovery mechanism. These features enable the model to simulate the strain-rate behavior exhibited by many metals over a wide range of temperatures. Over the past few years, this model has been modified and expanded by Bammann (3). This report only covers the material model in NIKE2D, the basic equations of which are summarized in Appendix A, which is based on an earlier version of theory which has twelve material constants. For discussion on formulation and numerical implementation ore can see Appendix $C$ in the 1986 NIKE2D manual (2).

In the following report, we describe the correct format to input data into the NIKE2D code, discuss the model's parameters as they apply to the NIKE2D code, and provides a procedure for determining model parameters from experimental data. 


\section{NIKE2D Input Format.}

In NIKE2D Bammann's model is material model \#11. The 1986 version of the NIKE2D manual has an incorrect format for inputting model parameters. Also, in the 1986 manual it is named incorrectly as Unified Creep Plasticity. The correct format is given here. (Note that when using the preprocessor MAZE, material \#11 does not correspond to material \#11 in NIKE2D, but rather to material \#11 in DYNA2D, a Temperature Dependent, Elastic-Plastic, Hydrodynamic model developed by Steinberg and Guinan.)

\section{Material \#11 Input Deck}

Card 1

Columns

Format

$1-5$

6-10

$11-80$

Card 2

$1-72$

Material identification number

Material type (11)

density (for dynamic analyses)

I5

I5

E10.0

Material identification

$12 \mathrm{~A} 6$

Card 3

$1-10$

$11-20$

$21-30$

$31-40$

$41-50$
Young's modulus

Poisson's ratio

Temperature (absolute)

$C_{13}$

$\chi, \quad(0 \leq \chi \leq 1)$
E10.0

E 10.0

E 10.0

E10.0

E10.0

Card 4

$\begin{array}{lll}1-10 & C_{1} & \mathrm{E} 10.0 \\ 11-20 & C_{2} & \mathrm{E} 10.0 \\ 21-30 & C_{3} & \mathrm{E} 10.0 \\ 31-40 & C_{4} & \mathrm{E} 10.0\end{array}$

Card 5

$1-10$

$11-20$

$21-30$

$31-40$
$C_{5}$

$\mathrm{C}_{6}$

$C_{7}$

$C_{8}$
E10.0

E 10.0

E10.0

E10.0

Card 6

1-10

$11-20$

21-30

$31-40$
$\mathrm{C}_{9}$

$C_{10}$

$C_{11}$

$C_{12}$
E10.0

E10.0

E10.0

E10.0 
Bammann $(3,4)$ has discussed in detail the physical interpretation of the material constants which occur in the current version of the theory, and has indicated how they should be determined. In what follows these issues are discussed within the context of using the model currently implemented into NIKE2D, and the effect of various parameters is exhibited with simple examples.

\section{Discussion of Material Constants in Bar'mann's Model.}

The constitutive model contains a number of temperature dependent parameters $(Y(\theta), V(\theta), f(\theta), k(\theta), g(\theta), h(\theta))$ whose functional dependence on temperature is prescribed in the code as an exponentialreciproc al. Each parameter depends on two material constants. Below, without going into the setails of the equations of the model, we discuss the role that each of the material constants play in describing different aspects of material behavior. The basic equations of the model are summarized in Appendix A. Refer to Appendix C of the 1986 NIKE2D manual (2) or one or more of the cited references of Bammann for a more thorough description of the constitutive equations of the model and its numerical implementation.

The temperature $(\theta)$ dependert material parameters are of the form: ${ }^{\dagger}$

and,

$$
Y(\theta)=C_{3} \exp \left(\frac{+C_{4}}{\theta}\right)
$$

$$
V(\theta), f(\theta), k(\theta), g(\theta), h(\theta): \quad \text { parameter }(\theta)=C_{n} \exp \left(\frac{-C_{n+1}}{\theta}\right),
$$

where $n=1,5,7,9,11$ (but $n \neq 3$ ). Note that the temperature dependence of these parameters is monotonic. The particular material behavior governed by the various constants is shown in the following:

$\begin{array}{lll}C_{3}, C_{4}: & Y(\theta) & \text { Rate-independent yield stress } \\ C_{1}, C_{2}: & V(\theta) & \text { Rate sensitivity of the yield stress } \\ C_{5}, C_{6:} & f(\theta) & \text { Transition to rate-dependent behavior } \\ C_{9}, C_{10:} & k(\theta) & \text { Hardening } \\ C_{11}, C_{12:} & g(\theta) & \text { Diffusion controlled "static" or } \\ C_{7}, C_{8}: & h(\theta) & \text { "thermal" recovery }\end{array}$

$\dagger$ The form of $Y(\theta)$ represents a recoding in a newer version NIKE2D to reflect the more recent work of Bammann. 
We now discuss the effect model's parameters (organized by material behavior).

\section{Temperature Dependence}

In the current implementation, temperature effects are due solely to the initial temperature state and from heat generation due to plastic deformation, which is considered purely adiabatic (see 2 below). The effect of the initial temperature (input on material card \#3 in NIKE2D) on stress is shown in figs. $1 \mathrm{a}$ and $1 \mathrm{~b}$. The current implementation of the model into NIKE2D cannot calculate stresses due to thermal expansion. The model also cannot accept temperature from either nodal cards or from nodal values determined by a heat transfer calculation. Modifications to NIKE2D will will have to be made before the model can be used in a coupled thermo-mechanical analysis.

The even numbered constants, $C_{2}, C_{4}, C_{6}, C_{8}, C_{10}$, and $C_{12}$, are associated with the temperature dependence in the model. It is always required to have a value for temperature (card 3 , columns 21-30). The values of $C_{1}, C_{3}, C_{5}, C_{7}, C_{9}, C_{11}$, will change depending on whether or not temperature dependence is included. For example, the constant $C_{3}$ represents a rate-independent yield stress with no temperature dependence. When temperature dependence is included $C_{3}$ and $C_{4}$ can be determined from the simple relationship below, and must both be changed:

and,

$$
C_{3}=Y_{1} \exp \left(\frac{-C_{4}}{\theta_{1}}\right)
$$

$$
C_{4}=\frac{\theta_{1} \theta_{2}}{\left(\theta_{2}-\theta_{1}\right)} \ln \left(\frac{Y_{1}}{Y_{2}}\right)
$$

where $Y_{1}$ and $Y_{2}$ represent the yield stress at two temperatures, $\theta_{1}$ and $\theta_{2}$ (see also section IV which follows).

\section{Heating Due to Plastic Work.}

The effect of heating due to plastic work is brought into the model through the constant $C_{13}$, where:

and

$$
\begin{aligned}
& C_{13}=\left(\frac{95}{\rho C_{\mathrm{v}}}\right), \\
& \rho=\text { density } \quad\left[\mathrm{kg} / \mathrm{m}^{3}\right],
\end{aligned}
$$

$$
C_{\mathrm{V}}=\text { specific heat } \quad\left[\mathrm{J} / \mathrm{kg} \cdot{ }^{\circ} \mathrm{K}\right] \text {. }
$$

A smaller value of specific heat will result in more adibatic heating as shown in fig. 2 . 


\section{Rate Effects.}

In the following, we omit an explicit discussion of the temperature dependent part of the responsc, but one should keep in mind that for each odd-numbered parameter discussed, the temperature dependence is governed by the subsequent even numbered parameter.

The model can be used to represent bilinear rate and temperature independent yield and hardening by specifying non-zero values for $C_{3}$, and $C_{9}$, only. Rateindependent recovery is included by specifying a non-zero value of $C_{7}$. Rate dependence is brought into the model through $C_{1}$ and $C_{5}$, with dynamic recovery being brought in through $C_{11}$. In NIKE2D, the rate of loading is linked to the nature of a load curve. For example, consider two load curves specifing the same amount of deformation in ten time steps: one with a time step size of one (nstep 10, delt 1) will give an order of magnitude higher strain-rate than one with a time step size of ten (nstep 10, delt 10). In the first case, the load history curve terminates at ten, whereas in the second case, it terminates at onc hundred. This is demonstrated in figs. $3 \mathrm{a}$ and $3 \mathrm{~b}$. This essentially means, that to increase the strain-rate you decrease the "time" for deformation. Rate effects of this type enter through the constitutive law, and NIKE2D can be run in the quasi-static mode. To account for inertial effects, the dynamic option must be flagged in the input deck, and a nonzero value for density must be specfied on material card \#1.

\section{Isotropic or Kinematic Hardening $\chi$.}

Analysis in which load paths are reversed may require an appropriate choice for the amounts of kinematic and isotropic hardening. The parameter $\chi$ (similar to the $\beta^{*}$ used in model \#3 in NIKE2D) can be set between 0.0 and 1.0 which represents hardening varying between kinematic and isotropic, respectively. An example of kinematic hardening is given in fig. $4 \mathrm{a}$ and isotropic hardening in fig. 4b. The analysis represents the response of a bar in which one end is given a positive displacement and then returned to zero displacement.

\section{Additional Examples.}

Consider the calculation of a uniaxial tension test shown in fig. 1a as a baseline, which uses the set of constants in section $V$ below (except for $C_{5}=1.02 \times 10^{-2}$ which enables the rate effects to be more clearly seen). Corresponding calculations were run while varying the value of individual constants. The effect of the constants on the stress response is illustrated in figs. 5-8:

- Fig. 5: inclusion of diffusion controlled static (or thermal) recovery, $g(\theta)$.

- Fig. 6: increasing dynamic recovery, $h(\theta)$.

- Fig. 7: decreasing $f(\theta)$, i.e., the rate at rate at which the transition to ratedependent behavior occurs.

- Fig. 8: decreasing the rate sensitivity of the yield stress, $V(\theta)$. 


\section{Determining Values for the Material Constants.}

In this section we discuss how to determine values for the material constants from experimental data. As indicated in the preceding sections, inelastic material behavior predicted by the model is governed by six temperature dependent functions, each of which depends on a pair of constants. The functional form of the temperature dependence of the parameters is not selected by the user, but their values may be affected by the specification of two constants each (n.b., section III above). For each parameter function, the corresponding even numbered constant affects the temperature dependence (setting the even numbered constant equal to zero makes a parameter temperature independent) while the preceding odd numbered constant effects the parameter's value whether the parameter is temperature dependent or not.

In order to determine an appropriate value for the two constants associated with a given parameter function, values for the function at two different temperatures need to be known. First we discuss how to determine values for each of the parameter functions from isothermal experimental data, and then we indicate how to obtain values for the two constants associated with a given parameter assuming ihat two values of the, parameter function are known.

\section{Determination of Values of the Parameters from Experimental Data.}

Bammann and co-workers $(3,4)$ have previously discussed in detail how values of the parameters in the most recent version of the theory can be determined from experimental data. Here ive briefly indicate how to determine values for the parameters occurring in the version of the theory coded into NIKE2D, and refer the reader to the references of Bammann et al. if more details are required. The parameters should be determined from tests which are essentially isothermal. In the following, it should be remembered that the value determined corresponds to one particular temperature, say $\theta_{0}$.

Consider a uniaxial stress test taking place at a constant temperature $\theta_{0}$ and a constant strain rate. Let $\sigma, \varepsilon$, and $\alpha$ denote the axial components of the stress, strain, and $\alpha_{i j}$ tensors, respectively (c.f., Appendix A). A reasonable approximation to the stress predicted by the model during loading may be expressed as

$$
\sigma \approx \alpha \pm\left(\kappa+Y\left(\theta_{0}\right)+V\left(\theta_{0}\right) \sinh ^{-1}\left(\frac{|\dot{\varepsilon}|}{f\left(\theta_{0}\right)}\right)\right),
$$

where the upper + sign corresponds to tensile loading while the lower - sign corresponds to comp.es sive loading (this neglects the elastic strain rate in comparison with the tota! strain rate). At initial yield, $\alpha$ and $\kappa$ are zero.

$Y\left(\theta_{0}\right)$ : Rate-Independent Part of the Yield Stress. At low strain rates the last term in the above expression for the stress is small in comparison with $Y\left(\theta_{0}\right)$. Moreover, at initial yield $\alpha$ and $\kappa$ are zero. So, the quasi-static (rate-independent) initial yield stress provides a value for $Y\left(\theta_{0}\right)$. 
$f\left(\theta_{0}\right) \sim$ Transition to Rate-Dependent Behavior.

$V\left(\theta_{0}\right) \sim$ Rate-Dependent Part of the Yield Stress. The last tern in the above expression for stress represents the rate dependent part of the initial yield stress. The parameter $f\left(\theta_{0}\right)$ represents the strain rate at which the behavior changes from being essentially rate independent to being rate dependent. The parameter $V\left(\theta_{0}\right)$ relates to the magnitude of the rate dependent part of the yield stress.

An appropriate value for $f\left(\theta_{0}\right)$ can be determined by running a sequence of uniaxial tests at varying strain rates (and a fixed temperature) and determining at what rate the yield stress begins to depend on the strain rate. The model's prediction of this transition takes place over roughly one decade of strain rate so extreme precision at identifying this transition value is not necessary. That is, the strain rate dependence predicted by the model is essentially zero for $\dot{\varepsilon}<f\left(\theta_{0}\right) / 10$, for $f\left(\theta_{0}\right) / 10<\dot{\varepsilon}$ $<f\left(\theta_{0}\right)$ a transition to rate dependent behavior takes place, and for $\dot{\varepsilon}>f\left(\theta_{0}\right)$ the initial yield stress is approximately linear in $\ln \dot{\varepsilon}$.

The magni.ude of the strain rate dependence is given by the parameter $V\left(\theta_{0}\right)$ which can be obtained from the yield stress $\sigma_{y}$ and the strain rate sensitivity parameter $m$, defined by

$$
m=\frac{\partial\left(\ln \sigma_{y}\right)}{\partial(\ln \dot{\varepsilon})}
$$

using the relationship

$$
V\left(\theta_{0}\right)=\sigma_{y} m
$$

which in many cases will be adequately represented by

$$
V\left(\theta_{0}\right)=Y\left(\theta_{0}\right) m \text {. }
$$

$\boldsymbol{k}\left(\theta_{0}\right)$ : Hardening. The theoretical model distinguishes between two facets of inelastic behavior, namely, hardening and recovery. Hardening is governed by the parameter $k\left(\theta_{0}\right)$. In the absence of recovery (i.e., $g\left(\theta_{0}\right)=h\left(\theta_{0}\right)=0$ ), or prior to substantial inelastic deformation (i.e., $\alpha \approx \kappa \approx 0$ ), the slope of the post-yield stessstrain curve is approximated by

$$
\frac{d \sigma}{d \varepsilon} \approx \frac{E k\left(\theta_{0}\right)}{E+k\left(\theta_{0}\right)}
$$

which can be further approximated in most cases, where $k\left(\theta_{0}\right)$ is much smaller than the elastic modulus $E$, by

$$
\frac{d \sigma}{d \varepsilon} \approx k\left(\theta_{0}\right)
$$

Thus, $k\left(\theta_{0}\right)$ can be obtained from the slope of the stress-strain curve just subsequent to yielding, or anywhere on the curve in cases where recovery is minimal. 
$g\left(\theta_{0}\right) \sim$ Static (or Thermal) Recovery.

$h\left(\theta_{0}\right) \sim$ Dynamic Recovery. In the absence of the terms which account for recovery, the model would essentially predict bilinear plasticity, in which the stressstrain curve had the slope $E$ prior to yield and the slope $k\left(\theta_{0}\right)$ after yield. The recovery terms bring in nonlinear inelastic response. In the case of monotonic loading the stress eventually "saturates" to the value

$$
\sigma_{\infty}=\sqrt{\frac{k\left(\theta_{0}\right) \dot{\varepsilon}}{g\left(\theta_{0}\right)+h\left(\theta_{0}\right) \dot{\varepsilon}}}+Y\left(\theta_{0}\right)+V\left(\theta_{0}\right) \sinh ^{-1}\left(\frac{|\dot{\varepsilon}|}{f\left(\theta_{0}\right)}\right) .
$$

At very low strain rates, the static recovery dominates, and the stress eventually approaches the value.

$$
\sigma_{\infty}=\sqrt{\frac{k\left(\theta_{0}\right) \dot{\varepsilon}}{g\left(\theta_{0}\right)}}+Y\left(\theta_{0}\right), \quad(\dot{\varepsilon} \rightarrow 0),
$$

which provides a way to determine $g\left(\theta_{0}\right)$. At higher strain rates, the dynamic recovery dominates, and the stress eventually approaches the value

$$
\sigma_{\infty}=\sqrt{\frac{k\left(\theta_{0}\right)}{h\left(\theta_{0}\right)}}+Y\left(\theta_{0}\right)+V\left(\theta_{0}\right) \sinh ^{-1}\left(\frac{|\dot{\varepsilon}|}{f\left(\theta_{0}\right)}\right), \quad(\dot{\varepsilon} \rightarrow \infty),
$$

which, in principal, provides a way to determine $h\left(\theta_{0}\right)$. In practice, however, it is difficult to have a test which maintains both high strain rates as well as isothermal conditions.

$\chi \sim$ Kinematic vs. Isotropic Hardening. Rather than having independent parameters in the constitutive equations for $\alpha$ and $\kappa$, as in the more general equations of the papers of Bammann et al., the code uses the same parameter functions but uses the constant $\chi$ to vary the hardening between kinematic $(\chi=0 \Rightarrow \kappa=0)$ and isotropic $\left(\chi=1 \Rightarrow \alpha_{i j}=0\right)$. The choice of which type of hardening is appropriate depends on both the loading path that the material is expected to undergo as well as the details of the stress strain curve which are desired to $\mathrm{s} \cdot \mathrm{matched}$ most closely. Clearly, if there is any reverse loading it is necessary to have a kinematic component of the hardening in order to predict realistic behavior. Another case in which the selection of $\chi$ can be exploited is to make the $\kappa$ part of the hardening reach saturation at a different rate the $\alpha_{i j}$ part. For example, choosing $\chi$ nearer to one, along with an appropriate value for $k\left(\theta_{0}\right), \kappa$ can be made to represent the rapid transient hardening which occurs near the "knee" of the stress-strain curve while $\alpha_{i j}$ represents the longer term part of the hardening. 


\section{Determination of Constants from Known Values of the Parameters.}

If a sufficiently large amount of data at various strain rates and temperatures is available, the optimum way to determine values for the constants occurring in the parameter functions is to perform a nonlinear least squares analysis. Below we indicate how to fit the parameter functions through two known values. The constants thus determined can then be used as initial guesses in a nonlinear least squares analysis, or in some cases they may by themselves suffice to adequately characterize the parameter functions.

Consider the parameter function $Y(\theta)$ which depends on the constants $C_{3}$ and $C_{4}$ (recall that $Y$ has a + exponential dependence on $1 / \theta$ whereas all the other parameters have a - exponential dependence). Suppose that the value of $Y$ is known at two different temperatures $\theta_{1}$ and $\theta_{2}$ to have the values $Y_{1}$ and $Y_{2}$, respectively. Then, in view of the functional form of $Y(\theta)$ indicated in section III part A, it follows that

and

$$
C_{4}=\frac{\theta_{1} \theta_{2}}{\left(\theta_{2}-\theta_{1}\right)} \ln \left(\frac{Y_{1}}{Y_{2}}\right)
$$

$$
C_{3}=Y_{1} \exp \left(\frac{-C_{4}}{\theta_{1}}\right)
$$

Similarly, for each of the other parameter functions $V(\theta), f(\theta), k(\theta), g(\theta)$, and $h(\theta)$ (which have a - exponential dependence on $1 / \theta$ ), if the value of a particular function is known at two temperatures $\theta_{1}$ and $\theta_{2}$ to have the values (say) $P_{1}$ and $P_{2}$, respectively, then the two constants associated with that parameter are determined from

and

$$
C_{n+1}=\frac{-\theta_{1} \theta_{2}}{\left(\theta_{2}-\theta_{1}\right)} \ln \left(\frac{P_{n}}{P_{n+1}}\right) \text {, }
$$

$$
C_{n}=P_{n} \exp \left(\frac{+C_{n+1}}{\theta_{1}}\right)
$$

for $n=1,5,7,9,11$ (but not for $n=3$ ). 


\section{An Example Set of Material Constants for Model \#11.}

A working set of twelve constants for the model are given for 21-6-9 stainless steel. The value of $C_{4}$ below represents a value appropriate for a newer version NIKE2D with a recoding of the $Y(\theta)$ parameter as indicated in section III above.

Values for 21-6-9 stainless steel

\begin{tabular}{|c|c|c|}
\hline Young's modulus & $2.00 \mathrm{e}+05$ & [MPa] \\
\hline Poisson's ratio & 0.30 & \\
\hline Temperature & 373 & {$\left[{ }^{\circ} \mathrm{K}\right]$} \\
\hline$\chi$ & 0.0 & \\
\hline$C_{13}$ & 0.268 & {$[\mathrm{Mkg}]$ or $\left[\mathrm{m}^{3} \cdot{ }^{\circ} \mathrm{K} / \mathrm{MJ}\right]$} \\
\hline$C_{1}$ & $5.58 \mathrm{e}+01$ & {$[\mathrm{MPa}]$} \\
\hline$C_{2}$ & $8.67 e+01$ & {$\left[{ }^{\circ} \mathrm{K}\right]$} \\
\hline$C_{3}$ & $1.67 e+01$ & {$[\mathrm{MPa}]$} \\
\hline$C_{4}$ & $4.68 \mathrm{e}+02$ & {$\left[{ }^{\circ} \mathrm{K}\right]$} \\
\hline$C_{5}$ & $1.02 e+04$ & {$\left[\mathrm{~s}^{-1}\right]$} \\
\hline$C_{6}$ & $3.48 \mathrm{e}+03$ & {$\left[{ }^{\circ} \mathrm{K}\right]$} \\
\hline$C_{7}$ & $4.72 \mathrm{e}-03$ & {$[1 / \mathrm{MPa}]$} \\
\hline$C_{8}$ & $2.55 \mathrm{e}+02$ & {$\left[{ }^{\circ} \mathrm{K}\right]$} \\
\hline$C_{9}$ & $1.81 \mathrm{e}+03$ & {$[\mathrm{MPa}]$} \\
\hline$C_{10}$ & $5.23 e+01$ & {$\left[{ }^{\circ} \mathrm{K}\right]$} \\
\hline$C_{11}$ & 0.0 & {$[1 / \mathrm{MPa} \cdot \mathrm{s}]$} \\
\hline$C_{12}$ & 1.0 & {$\left[{ }^{\circ} \mathrm{K}\right]$} \\
\hline
\end{tabular}

\section{Acknowledgement}

The authors would like to thank Professor George C. Johnson, University of California, Berkeley, for many useful discussions regarding the behavior and implementation of this material model. 


\section{REFERENCES}

1. Bammann, D. J., "An Internal Variable Model of Viscoplasticity," Int. J. Eng. Sci., $11,(1984), 1041-1053$.

2. Hallquist, J. O., "NIKE2D, A Vectorized Implicit, Finite Defonnation, Finite Element Code for Analyzing the Static and Dynamic Response of 2-D Solids with Interactive Rezoning and Graphics," UCID 19677, Rev. 1,1986

3. Bammann, D. J., "Modeling the Temperature and Strain Rate Dependent Large Deformation of Metals," Applied Mechanici Reviews, Proceedings of the 11 th U. S. National Congress of Applied Mechanics, Tucson, AZ, May 1990.

4. Bammann, D. J., Unpublished manuscripts. 


\section{Appendix A. Basic Equations of Bammann's Model.}

Let,

$$
\underset{\sim}{\tau} \equiv \underset{\sim}{R^{\mathrm{T}}} \underset{\sim}{\stackrel{R}{R}}
$$

denote the rotated Cauchy stress,

$$
\underline{\xi} \equiv \underline{\tau}^{\prime}-\underline{\alpha}^{\prime}
$$

where ( $)^{\prime}$ denotes the deviatoric part of ( ), and

$$
\delta \equiv \underset{\sim}{R} \underset{\sim}{\mathrm{T}} \underset{\sim}{R}
$$

denote the rotated stretching rate, where

$$
\underset{\sim}{D}=\operatorname{sym}\left(\nabla_{\sim}\right),
$$

is the rate of deformation tensor. A subscript $p$ refers to the plastic part of the deformation. The stress response is given by

$$
\dot{\tau}=\frac{E v}{(1+v)(1-2 v)} \operatorname{tr}(\underset{\delta}{\delta}) \underset{\sim}{1}+\frac{E}{(1+v)}\left(\underline{\delta}-\underline{\delta}_{p}\right),
$$

and the "flow function" is given by

$$
\phi=\sqrt{\frac{3}{2} \underset{\sim}{\xi} \underline{\xi}}-\kappa-Y(\theta)-V(\theta) \sinh ^{-1}\left(\frac{|\underline{\delta}|}{f(\theta)}\right) .
$$

If a trial stress state is inside the "flow surface" (i.e., $\phi \leq 0$ ) then no plastic deformation occurs, and

$$
\begin{gathered}
\delta_{p}=0, \\
\dot{\alpha}=-g(\theta) \frac{|\underline{\alpha}| \underline{\alpha}}{(1-\chi)}, \\
\dot{\kappa}=-g(\theta) \frac{\kappa^{2}}{\chi} .
\end{gathered}
$$


On the other hand, if the trial stress state falls outside the flow surfact (i.e., $\phi>0$ ) then

$$
\begin{aligned}
& \delta_{p}=f(\theta) \sinh \left[\frac{\mid \underline{\mid \xi}-\kappa-Y(\theta)}{V(\theta)}\right] \frac{\underline{\xi}}{|\underline{\xi}|},
\end{aligned}
$$

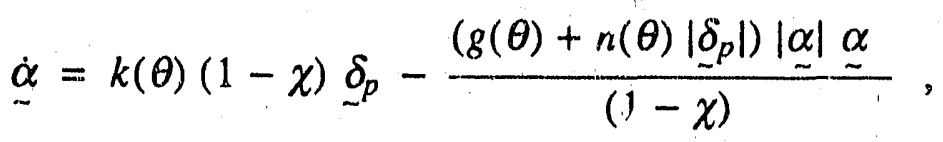

$$
\begin{aligned}
& \dot{\kappa}=\dot{k}(\theta) \chi \underline{\sigma}_{p} \mid-\frac{\left(g(\theta)+h(\theta) ! \delta_{p} \mid\right) \kappa^{2}}{\chi},
\end{aligned}
$$

and adiabatic heating esults in,

$$
\dot{\theta}=C_{13}\left(\underline{\tau} \cdot \underline{\delta}_{p}\right)
$$

Note that even inside the flow surface, if prior plastic deformation has occurred then the internal variables $\alpha_{i j}$ and $\kappa$ may change. This is meant to allow for "recovery" even if no plastic deformation is occurring. These changes affect the value of the flow function, and hence the stress at which re-yielding may occur, but they do not affect the stress-strain behavior within the flow surface. This contrasts with more classical elastic-plastic models for which the elastic region (i.e., within the yield surface) implies that no inelastic changes occur. For this reason, Binmann's reference to deformations ocurring inside the flow surface as "elastic" is sorıewhat misleading. 


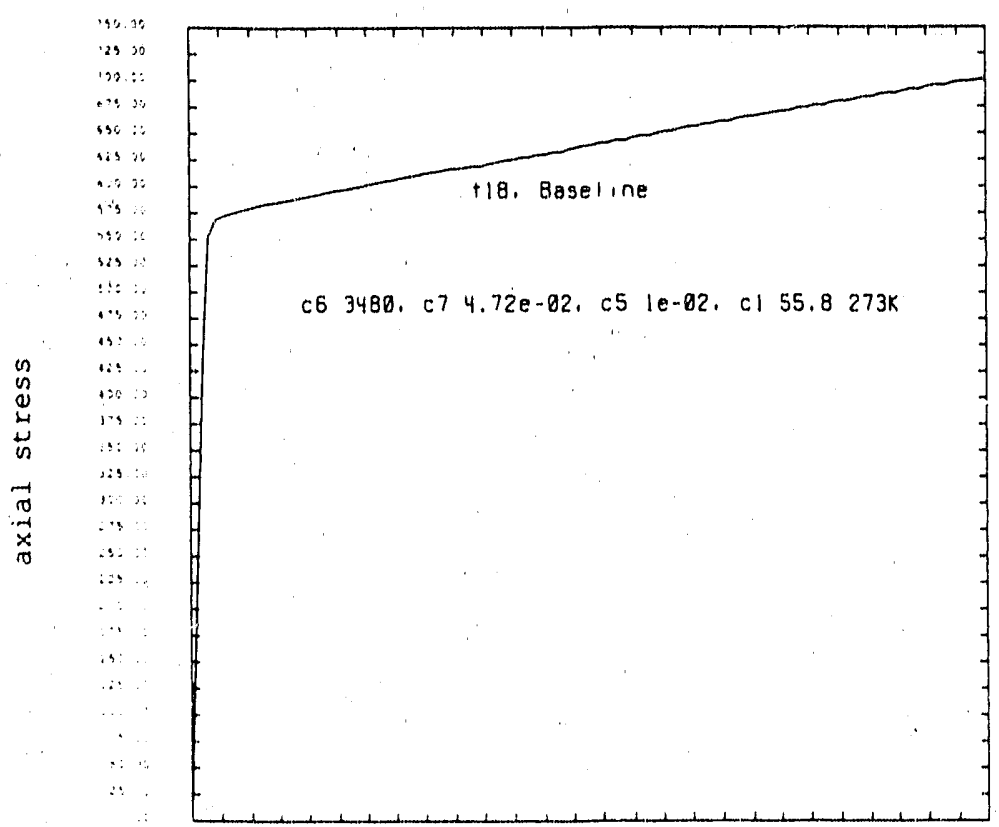

axial strain

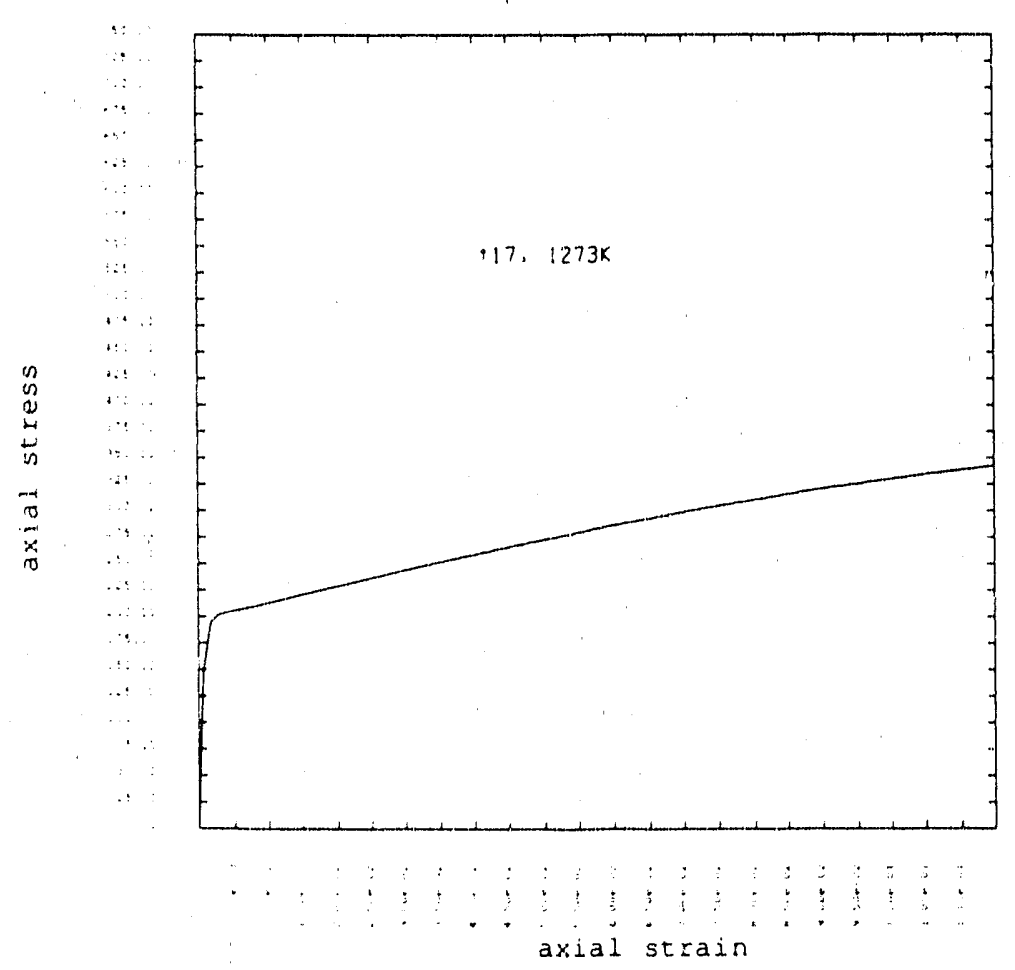

Fig. 1a. Baseline calculation at $273^{\circ} \mathrm{K}$ using constants given in section $\mathrm{V}$ except for $C_{5}$ which was changed to $1.0 \times 10^{-2} \mathrm{~s}^{-1}$ to capture rate effects.
Fig. 1b. Temperature raised to $1273^{\circ} \mathrm{K}$. 


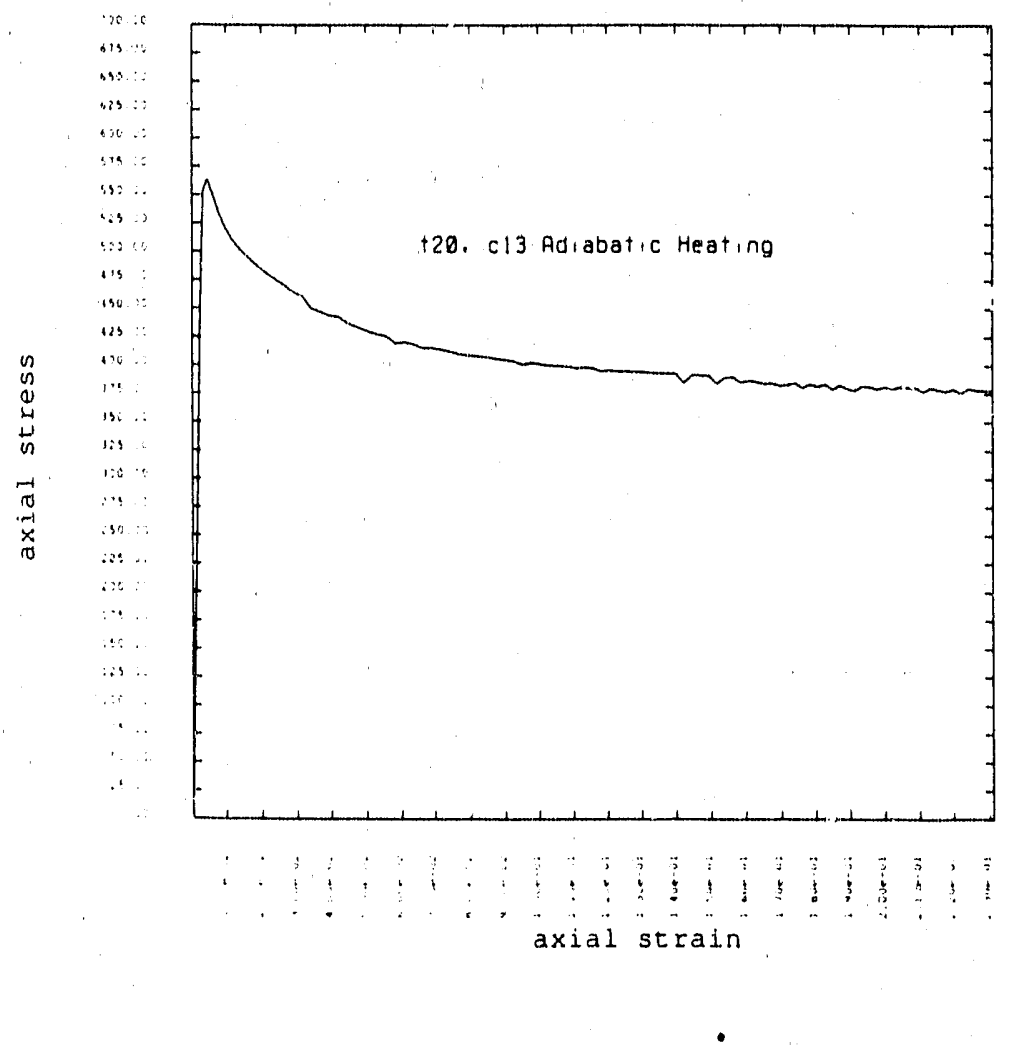

Fig. 2. Adiabatic heating due to a lower value of specific heat. 


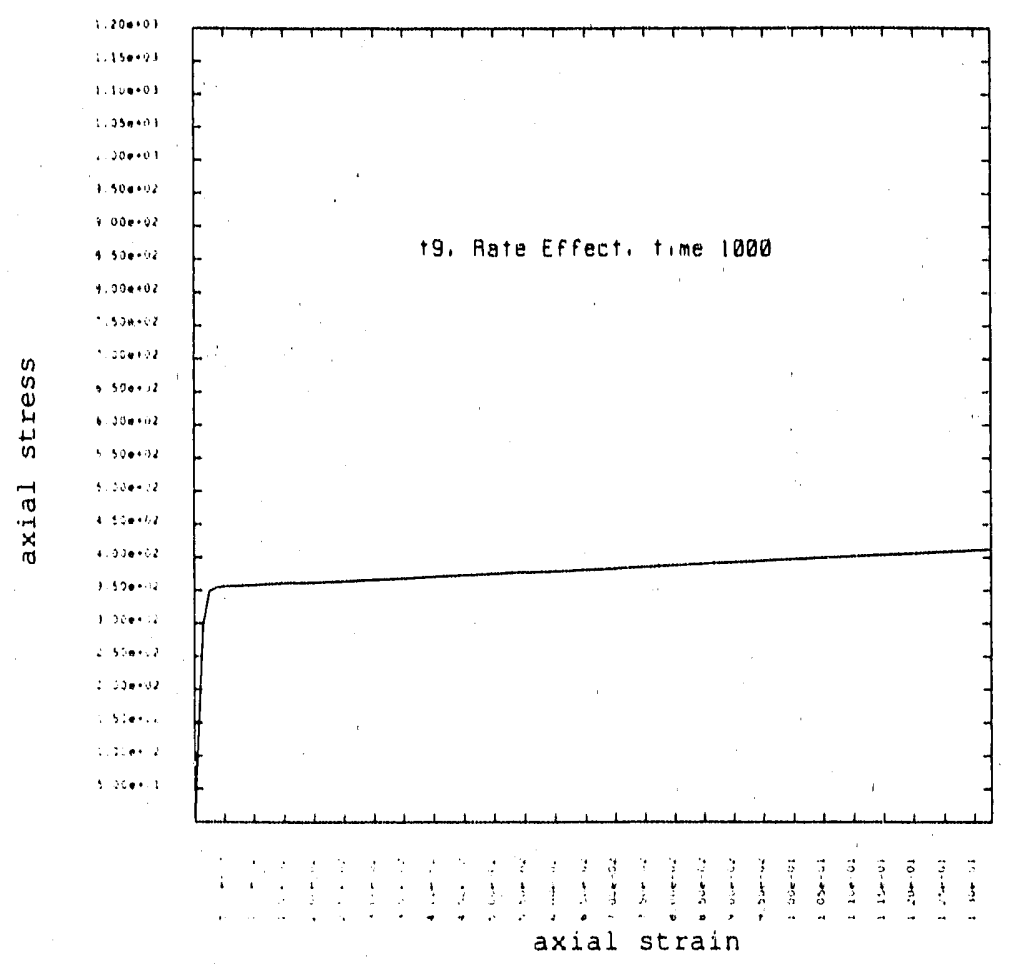

Fig. 3a. Strain rate of approx. $1.30 \times 10^{-4} \mathrm{~s}^{-1}$

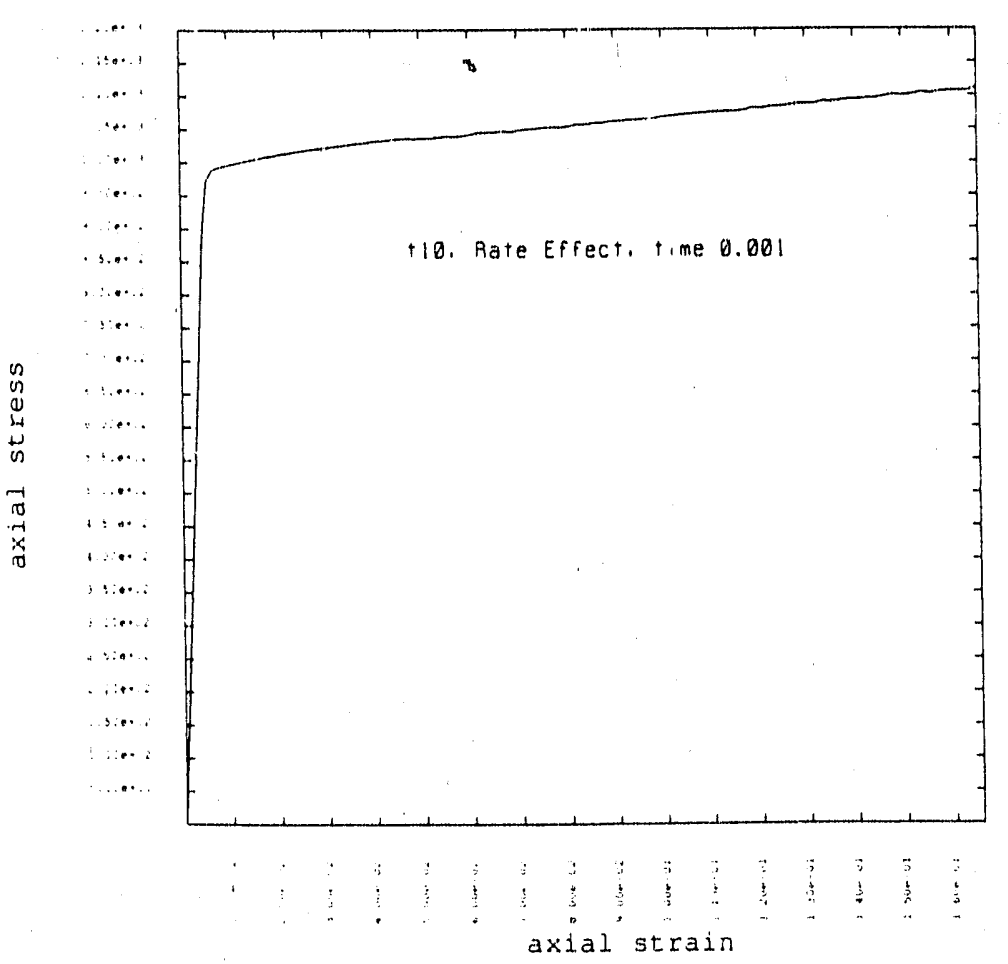

Fig. 3b. Strain rate of approx. $1.60 \times 10^{+1} \mathrm{~s}^{-1}$. 

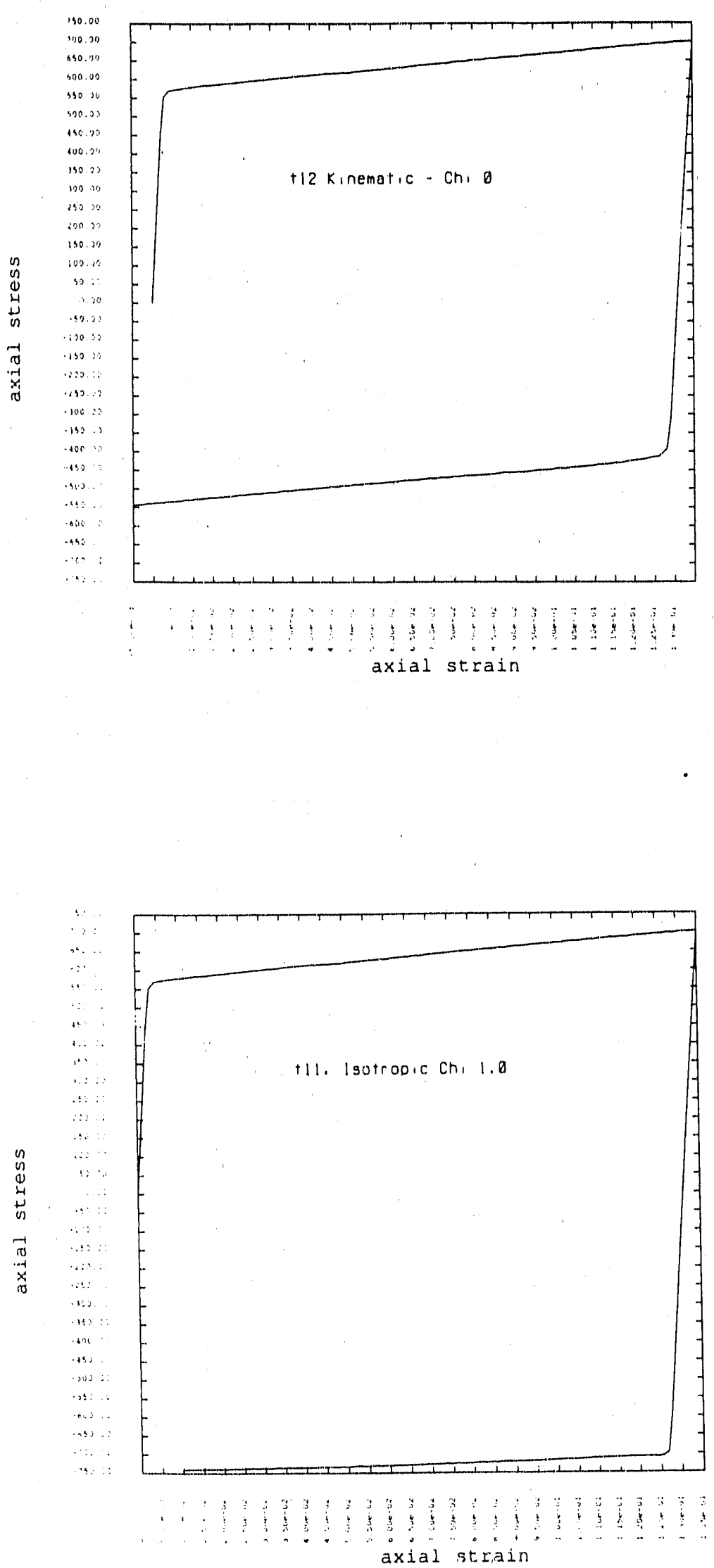

Fig. 4a. Kinematic hardening with $\chi=0.0$.
Fig. 4b. Isotropic hardening with $\chi=1.0$. 


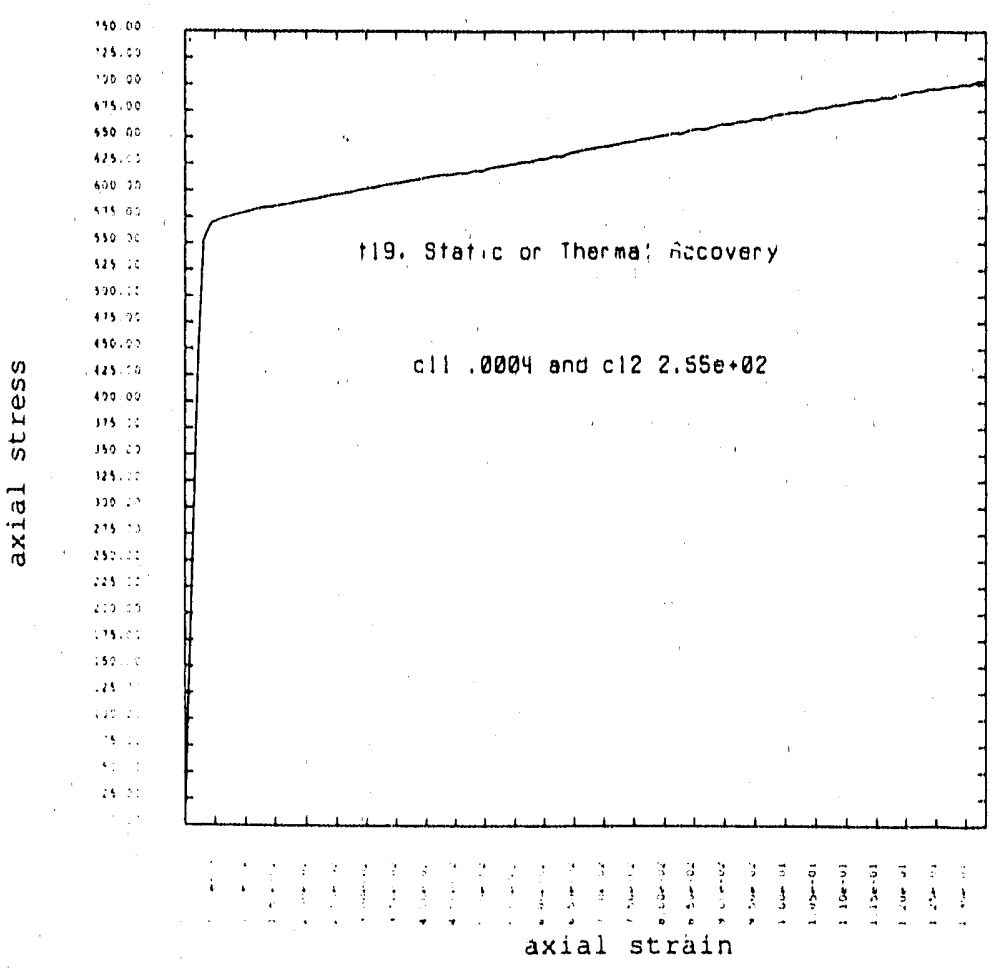

Fig 5. Inclusion of diffusion controlled or static recovery, $g(\theta)$.

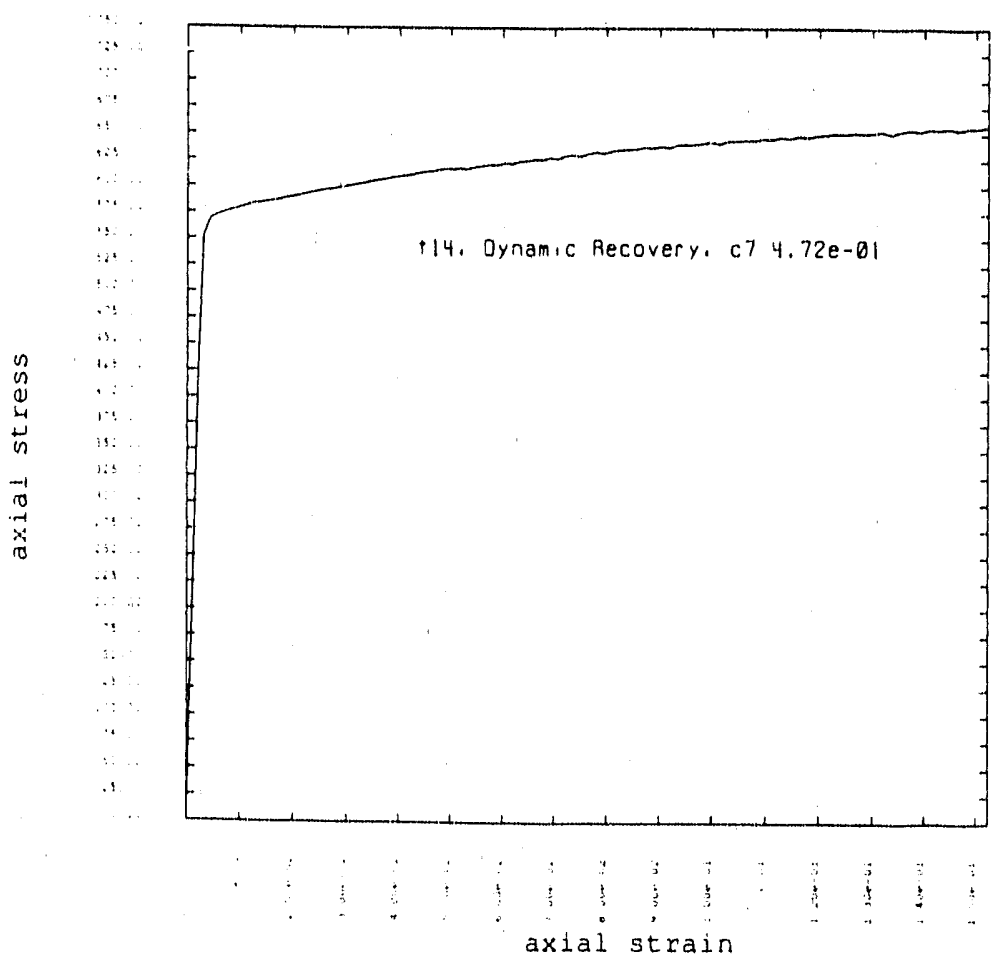

Fig. 6. Increasing dynamic recovery, $h(\theta)$. 


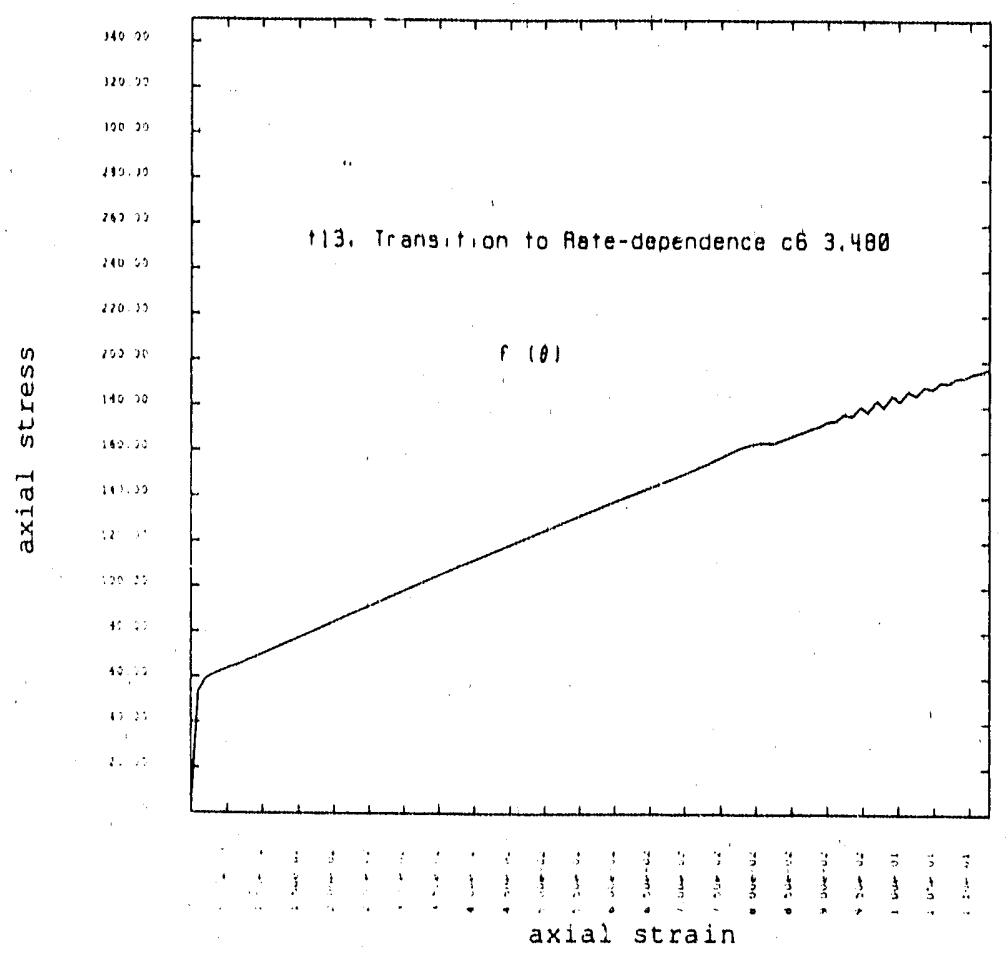

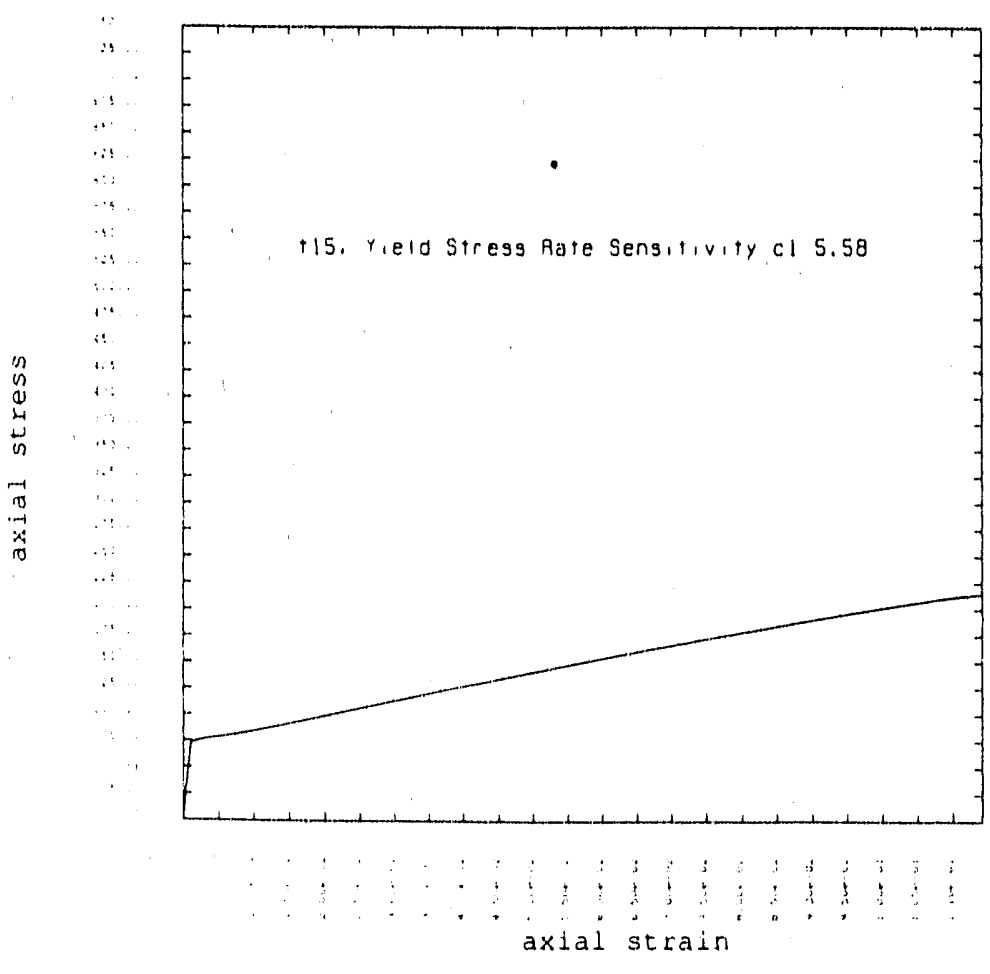

Fig. 7. Decreasing $f(\theta)$, i.e., the rate at which the transition to rate-dependent behavior occurs.
Fig. 8. Decreasing the rate sensitivity of the yield stress, $V(\theta)$. 

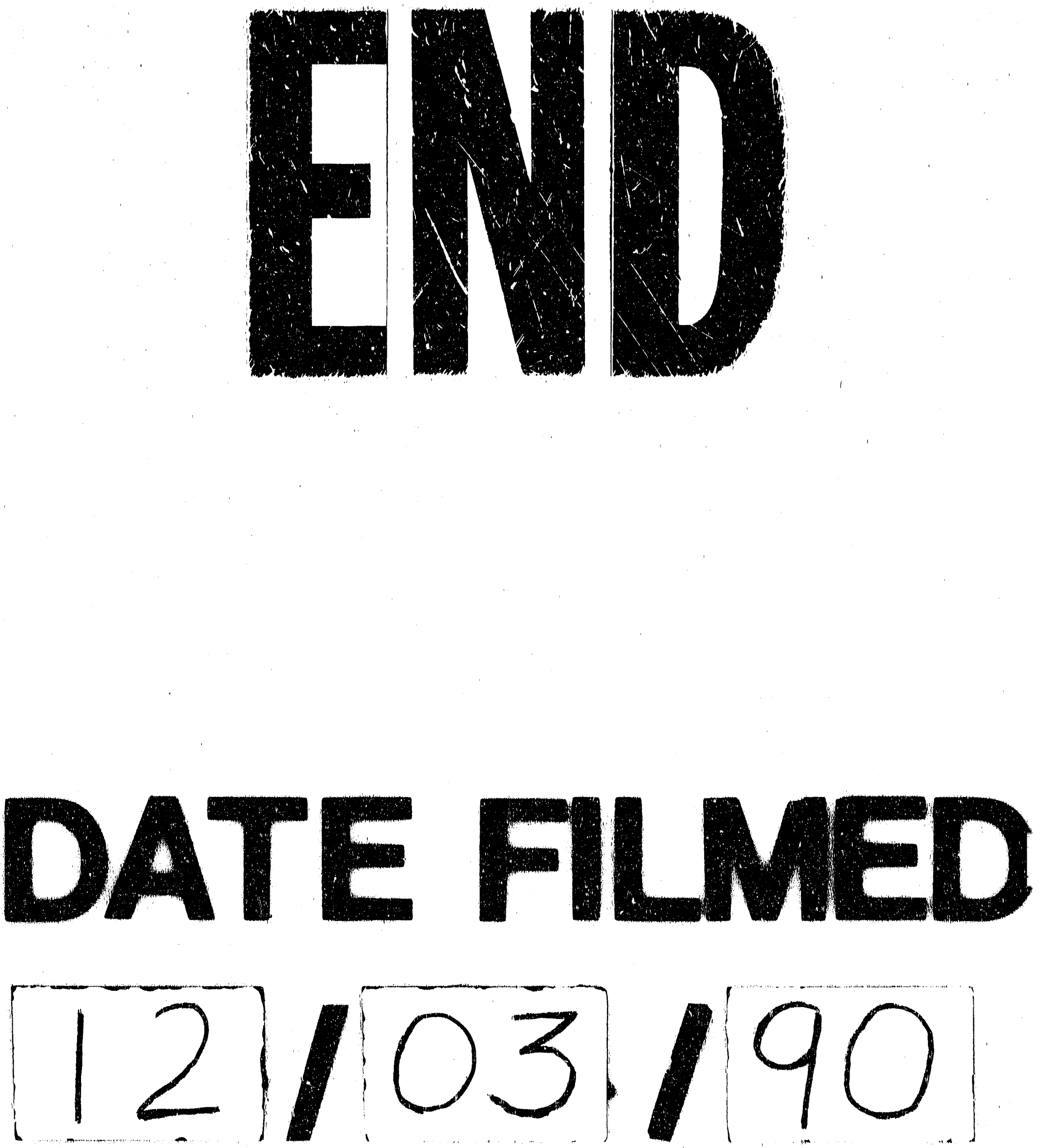
